\title{
Luoyutong Treatment Promotes Functional Recovery and Neuronal Plasticity after Cerebral Ischemia-Reperfusion Injury in Rats
}

\author{
Ning-qun Wang, ${ }^{1,2}$ Li-ye Wang, ${ }^{3}$ Hai-ping Zhao,, ${ }^{1,4}$ Ping Liu, ${ }^{1,5}$ Rong-liang Wang, ${ }^{1,4}$ \\ Jue-xian Song, ${ }^{5}$ Li Gao, ${ }^{5}$ Xun-ming Ji, ${ }^{4}$ and Yu-min Luo ${ }^{1,4}$ \\ ${ }^{1}$ Cerebrovascular Diseases Research Institute, Xuanwu Hospital, Capital Medical University, Ministry of Education, \\ 45 Changchun Street, Beijing 100053, China \\ ${ }^{2}$ Department of Traditional Chinese Medicine, Xuanwu Hospital, Capital Medical University, Ministry of Education, \\ 45 Changchun Street, Beijing 100053, China \\ ${ }^{3}$ Dongfang Hospital, Beijing University of Chinese Medicine, Beijing 100078, China \\ ${ }^{4}$ Key Laboratory of Neurodegenerative Diseases (Capital Medical University), Ministry of Education, \\ 45 Changchun Street, Beijing 100053, China \\ ${ }^{5}$ Department of Neurology, Xuanwu Hospital, Capital Medical University, Ministry of Education, 45 Changchun Street, \\ Beijing 100053, China
}

Correspondence should be addressed to Yu-min Luo; yumin111@ccmu.edu.cn

Received 9 September 2015; Revised 5 November 2015; Accepted 15 November 2015

Academic Editor: Shan-Yu Su

Copyright (C) 2015 Ning-qun Wang et al. This is an open access article distributed under the Creative Commons Attribution License, which permits unrestricted use, distribution, and reproduction in any medium, provided the original work is properly cited.

\begin{abstract}
Luoyutong (LYT) capsule has been used to treat cerebrovascular diseases clinically in China and is now patented and approved by the State Food and Drug Administration. In this retrospective validation study we investigated the ability of LYT to protect against cerebral ischemia-reperfusion injury in rats. Cerebral ischemia-reperfusion injury was induced by middle cerebral artery occlusion followed by reperfusion. Capsule containing LYT (high dose and medium dose) as treatment group and Citicoline Sodium as positive control treatment group were administered daily to rats $30 \mathrm{~min}$ after reperfusion. Treatment was continued for either 3 days or 14 days. A saline solution was administered to control animals. Behavior tests were performed after 3 and 14 days of treatment. Our findings revealed that LYT treatment improved the neurological outcome, decreased cerebral infarction volume, and reduced apoptosis. Additionally, LYT improved neural plasticity, as the expression of synaptophysin, microtubule associated protein, and myelin basic protein was upregulated by LYT treatment, while neurofilament 200 expression was reduced. Moreover, levels of brain derived neurotrophic factor and basic fibroblast growth factor were increased. Our results suggest that LYT treatment may protect against ischemic injury and improve neural plasticity.
\end{abstract}

\section{Introduction}

Stroke is one of the leading causes of death worldwide and causes long-term disability. It has a significant impact upon health, well-being, and social interactions. Despite this, the available treatments remain limited and unsatisfactory and there is a considerable demand for novel therapies [1-3]. The use of Traditional Chinese Medicines (TCMs) in treating cerebral ischemia injury has increased in recent years and the regulatory pathways targeted by these medicines have been investigated [4-6]. A special group of medicinal plants and animals used in TCMs have been widely administered as patented Chinese medications to manage the symptoms of stroke, such as spasticity, altered muscle tone, and motor neuron excitability [7-10].

Neuronal plasticity involves changes in intracerebral structure and function in both gray matter and white matter. Gray matter consists mainly of neuronal cell bodies and unmyelinated axons. Within the gray matter, synaptic distribution and density can be measured by expression of the 
TABLE 1: LYT ingredients.

\begin{tabular}{|c|c|c|c|c|}
\hline Ingredients (Latin name) & Family & Part used & Processing & Amount used \% \\
\hline \multicolumn{5}{|l|}{ Plants } \\
\hline Astragalus membranaceus (Fisch.) Bge. & Leguminosae & Dried root & Extraction & 18.159 \\
\hline Ligusticum chuanxiong Hort. & Umbelliferae & Root and rhizome & Extraction & 9.079 \\
\hline Spatholobus suberectus Dunn & Papilionaceae & Rattan and stem & Extraction & 13.681 \\
\hline \multicolumn{5}{|l|}{ Insects } \\
\hline Pheretima vulgaris Chen & Guaibasauridae & Dried body & Farina & 27.238 \\
\hline Whitmania pigra Whitman & Hirundinidae & Dried body & Farina & 13.681 \\
\hline Buthus martensii Karsch & Buthidae & Dried body & Farina & 5.472 \\
\hline Scolopendra subspinipes mutilans L. Koch & Psittacidae & Dried body & Farina & 4.477 \\
\hline Bombyx mori Linnaeus & Bombycidae & Dried body & Farina & 8.208 \\
\hline
\end{tabular}

synaptic vesicle protein synaptophysin, while dendrites can be visualized using microtubule associated protein (MAP-2) as a marker. White matter is composed mainly of myelinated axons and myelin-producing oligodendrocytes as well as other glial cells. Myelin basic protein (MBP) is a marker of myelin and neurofilament 200 (NF200) is expressed in myelinated axons. The effect of stroke on gray matter has been well studied in the past, but more attention has been paid recently to white matter injury following stroke [11].

Luoyutong (LYT) capsule contains eight active TCM ingredients (Table 1) and is patented and approved by the State Food and Drug Administration in China. It has been used clinically for the treatment of acute and chronic cerebrovascular diseases. Previous studies showed it has a therapeutic effect of stroke $[12,13]$. Our preliminary results suggest LYT plays a protective role in cerebral ischemia-reperfusion injury of rats [14]; however, the mechanism underlying the therapeutic effects of LYT in cerebrovascular diseases remains undefined. To address this, in this retrospective validation study, we investigated the protective effect and underlying mechanisms of LYT treatment in a rat model of cerebral ischemia-reperfusion injury.

\section{Methods}

2.1. Drug and Preparation. LYT (505 Pharmaceutical Co. Ltd., Lot number: 960815, Xianyang, Shanxi, China) was in the form of a dried superfine powder $(\leq 10 \mu \mathrm{m})$ composed of eight ingredients (Table 1), which were ground using a micronizer. LYT powder was prepared as a capsule, which was authenticated and standardized based on marker compounds in the Chinese Pharmacopoeia (Committee, 2005). The ingredients of the LYT capsule were carefully analyzed and quality-controlled. One gram of capsule is equivalent to $2.68 \mathrm{~g}$ of crude drug. The powder was dissolved in saline $(0.08 \mathrm{~g} / \mathrm{mL})$ and stored at $4^{\circ} \mathrm{C}$ until subsequent use. Citicoline Sodium (CS) capsule (QILU Pharmaceutical Group Co. Ltd., Lot number: H20020220, Jinan, Shandong, China) was used clinically for cerebral ischemia, cerebral hemorrhage, and dementia because of its repair effect in recovery phase. Citicoline Sodium (CS) capsules were diluted in saline to a final concentration of $2 \mathrm{mg} / \mathrm{mL}$ as a positive control treatment group.
2.2. Animals. Male Sprague-Dawley rats weighing 280-300 g were purchased from Vital River Laboratory Animal Technology Co. Ltd. (Beijing, China). Animals were housed in an environmentally controlled room at $22 \pm 2^{\circ} \mathrm{C}$, with a $12 \mathrm{~h} / 12 \mathrm{~h}$ light/dark cycle and were allowed free access to food and water throughout the entire study. The study was approved by the Institutional Animal Care and Use Committee of Capital Medical University and was in accordance with the principles outlined in the National Institutes of Health Guide for the Care and Use of Laboratory Animals.

2.3. Rat Model of Cerebral Ischemia-Reperfusion. Focal cerebral ischemia was induced in rats as previously described [15]. Briefly, rats were anesthetized with enflurane, and the right common carotid artery, the external carotid artery (ECA), and the internal carotid artery (ICA) were exposed. A 4-0 suture (diameter, $0.26 \mathrm{~mm}$ ) with a blunted tip coated with poly-L-lysine was gently advanced into the ICA through the ECA. The suture was advanced $18-20 \mathrm{~mm}$ (reaching the origin of the right middle cerebral artery) beyond the carotid artery bifurcation. To allow reperfusion, the suture was slowly withdrawn after $1.5 \mathrm{~h}$ of middle cerebral artery occlusion (MCAO). Regional cerebral blood flow $(0.5 \mathrm{~mm}$ anterior and $5.0 \mathrm{~mm}$ lateral to bregma) was monitored using laser Doppler flowmetry (PeriFlux System 5000, Perimed, Stockholm, Sweden) to ensure the occurrence of ischemia by MCAO. Rectal temperature was maintained at $37.0^{\circ} \mathrm{C}$ during and after surgery with a temperature-controlled heating pad (CMA 150 Carnegie Medicine, Sweden). Operations in the sham group were performed using the same surgical procedures, excepting the occlusion of the carotid arteries. All animals were housed in an air-conditioned room at $22 \pm 2^{\circ} \mathrm{C}$ after recovering from anesthesia.

2.4. Grouping and Treatment. In total, 90 rats were randomly divided into five groups: sham group, MCAO group, medium dose $(0.4 \mathrm{~g} / \mathrm{kg})$ of LYT (LYTM), high dose $(0.8 \mathrm{~g} / \mathrm{kg})$ of LYT $(\mathrm{LYTH})$, and CS $(0.1 \mathrm{~g} / \mathrm{kg})$. Each group was divided into two subgroups, one treated for 3 days and the other for 14 days. Each subgroup contained nine animals; six were used for 2,3,5-triphenyltetrazolium chloride (TTC) staining to evaluate brain infarct and swelling volume and three rats were allocated for histological and Western blot analyses. 


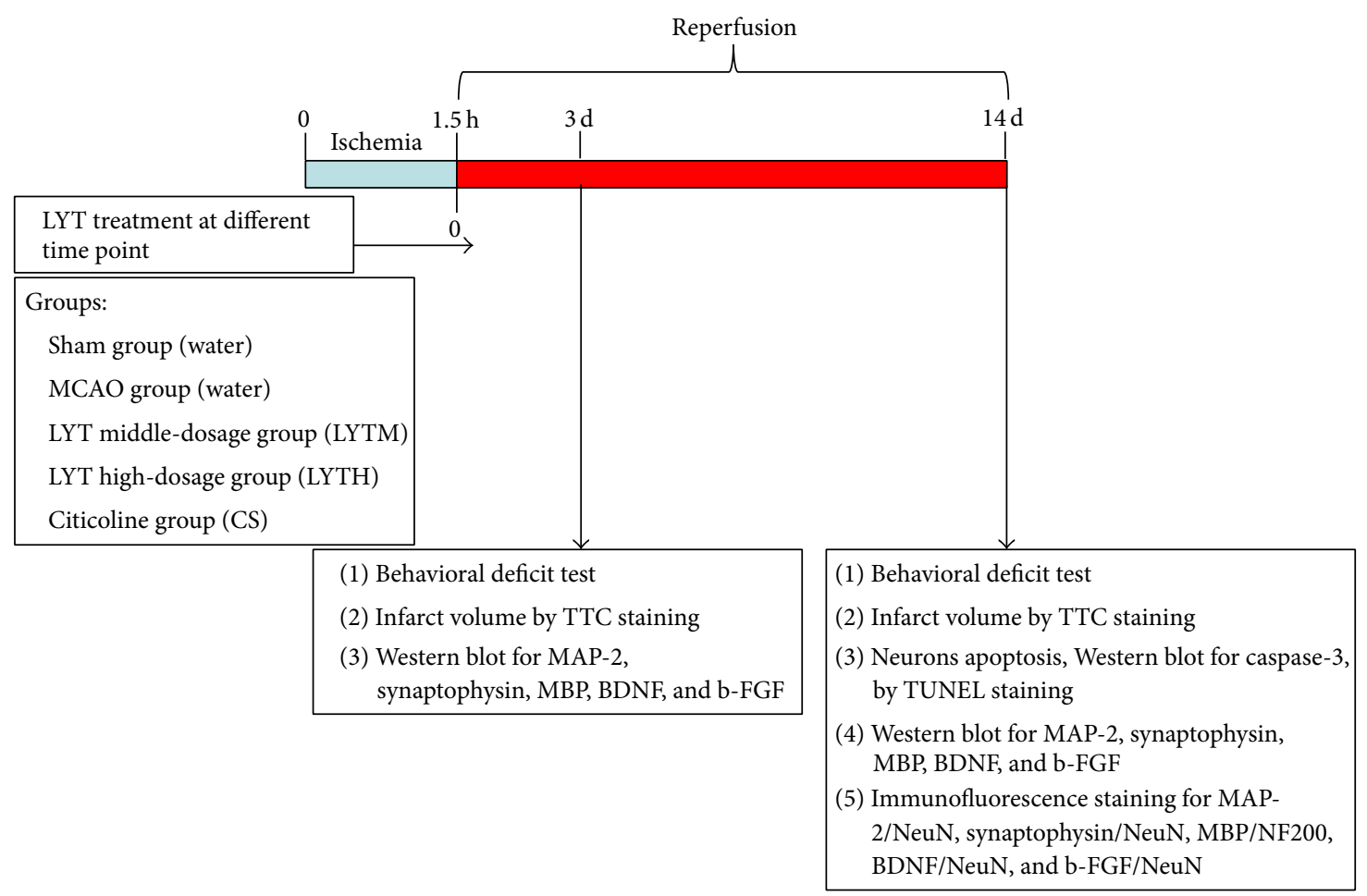

FIGURE 1: Schematic representation of the experimental procedures. (Luoyutong: LYT; middle cerebral artery occlusion: MCAO; 2,3,5triphenyltetrazolium chloride: TTC; microtubule associated protein: MAP-2; myelin basic protein: MBP; brain derived neurotrophic factor: BDNF; basic fibroblast growth factor: b-FGF; neuron-specific nuclear protein: NeuN; neurofilament 200: NF200).

A drug solution $(2 \mathrm{~mL})$ was administered by gavage and was calculated according to the body surface area based on the daily clinical dosage recommended for humans. Treatment started $30 \mathrm{~min}$ after reperfusion and was administered once a day for 3 or 14 days. Animals in the sham and MCAO groups received $2 \mathrm{~mL} 0.9 \% \mathrm{NaCl}$ in the same manner. The experimental design is illustrated in Figure 1.

2.5. Evaluation of Motor Performance. Behavioral tests were performed blindly by a trained investigator to eliminate bias. Three tests were carried out to evaluate various aspects of neurological function. (1) The first one is Longa's score test [15], where a normal score is 0 and the maximum score is 5 . (2) The second one is the $0-12$ neurological score test, which included the postural reflex and forelimb placing test, graded on a scale of 0 to 12 . The postural reflex test examined the upper body posture while the animal was suspended by the tail and the forelimb placing test evaluated the response of the forelimb to visual, tactile, and proprioceptive stimuli. (3) The third one is the modified foot fault test $[16,17]$ which recorded the number of times the forelimb was misplaced, causing the rat to fall through the grid.

2.6. Measurement of Infarct Volume. The animals were sacrificed 3 or 14 days after reperfusion in a carbon dioxide chamber and the brains were quickly removed and sectioned into six consecutive coronal slices of $2 \mathrm{~mm}$ thickness. The slices were stained by immersing in 2\% TTC for $30 \mathrm{~min}$ at $37^{\circ} \mathrm{C}$, followed by fixation in $8 \%$ formalin. The border between infarcted and noninfarcted tissues was outlined with an image analysis system. The area of infarction was measured by subtracting the area of the nonlesioned ipsilateral hemisphere from that of the contralateral hemisphere based on Swanson's method [18]. The infarct volume was calculated as follows: $100 \% \times$ (contralateral hemisphere volume nonlesioned ipsilateral hemisphere volume)/contralateral hemisphere volume. The swelling volume was calculated as follows: $100 \% \times$ (ipsilateral hemisphere volume - contralateral hemisphere volume)/contralateral hemisphere volume.

\subsection{Terminal Deoxynucleotidyl Transferase dUTP Nick End} Labeling (TUNEL). Rats were euthanized 14 days after reperfusion with intraperitoneal injections of chloral hydrate $(300 \mathrm{mg} / \mathrm{kg}$ ) and were perfused transcardially with $4 \% \mathrm{w} / \mathrm{v}$ paraformaldehyde in phosphate-buffered saline (PBS). The brains were dehydrated in 30\% sucrose in $4 \%$ formaldehyde in PBS. Frozen brains were sectioned coronally. Apoptotic cell death was detected using the In Situ Cell Death Detection Kit, POD (Roche, San Francisco, CA, USA), according to the manufacturer's instructions.

2.8. Western Blotting Analysis. The forward fontanelle, cut at a coronal slice thickness of $2 \mathrm{~mm}$ from the optic chiasma, was harvested 3 or 14 days after reperfusion. Samples were homogenized in lysis buffer $(50 \mathrm{mM}$ Tris- $\mathrm{HCl}, \mathrm{pH}$ $7.5,100 \mathrm{mM} \mathrm{NaCl}$, and $1 \%$ Triton X-100) containing protease inhibitors (aprotinin, leupeptin, phenylmethylsulfonyl 


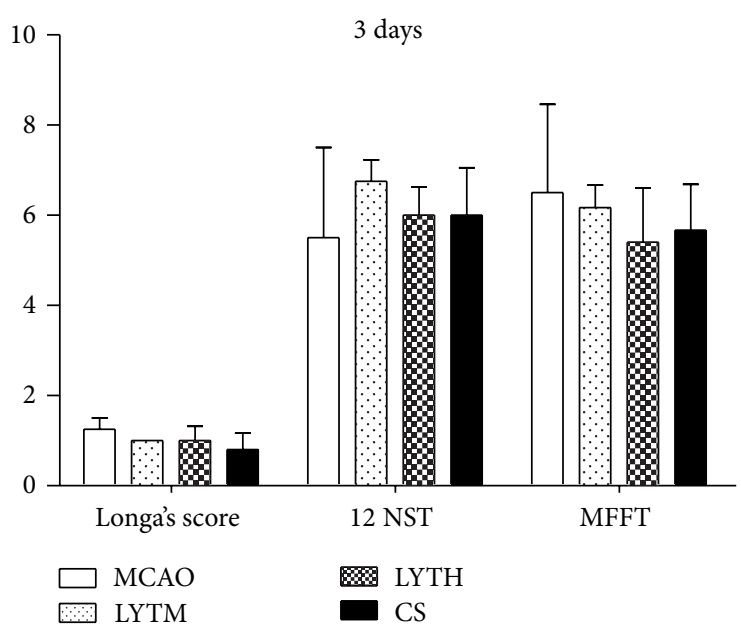

(a)

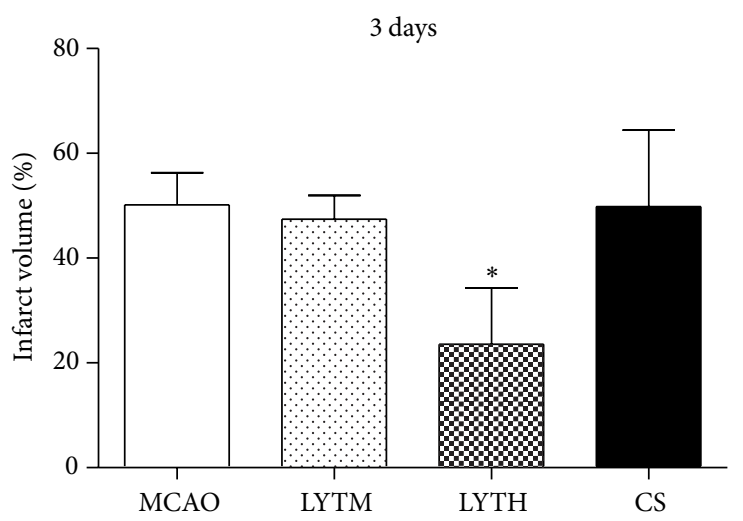

(c)

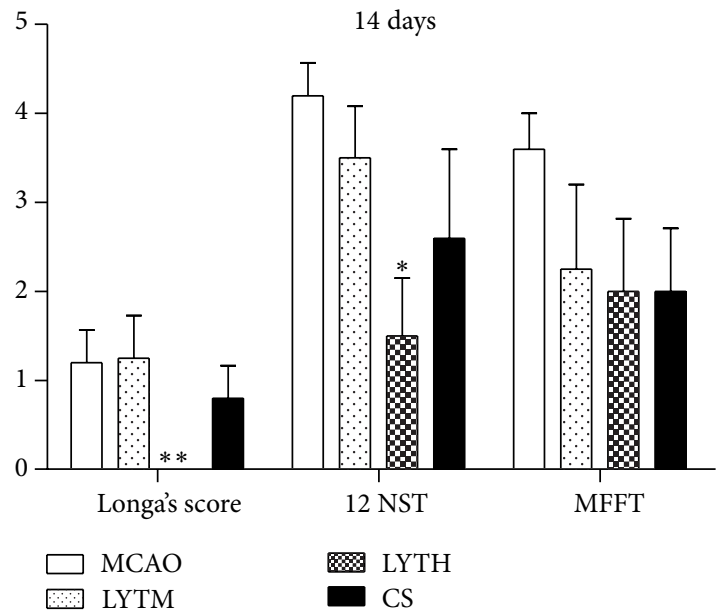

(b)

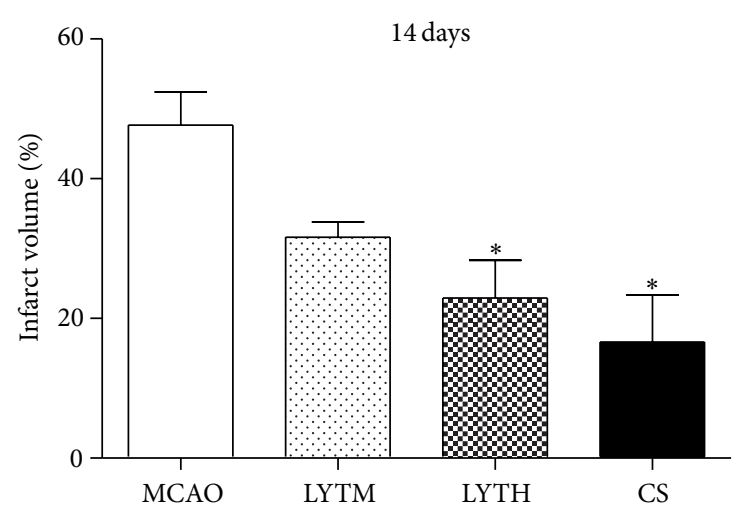

(d)

FiguRE 2: LYT (Luoyutong) decreases infarct volume and improves neurological function in rats following ischemic reperfusion. (a) Longa's score, $0-12$ neurological score test, and modified foot fault test of MCAO (middle cerebral artery occlusion), LYTM (medium dose (0.4 g/kg) of LYT), LYTH (high dose ( $0.8 \mathrm{~g} / \mathrm{kg}$ ) of LYT), and CS (Citicoline Sodium) 3 days after MCAO. (b) Longa's score, 0-12 neurological score test, and modified foot fault test of MCAO, LYTM, LYTH, and CS 14 days after MCAO. (c) Infarct volume (\%) of MCAO, LYTM, LYTH, and CS 3 days after MCAO. (d) Infarct volume (\%) of MCAO, LYTM, LYTH, and CS 14 days after MCAO; ${ }^{*} P<0.05$ versus $M C A O$ and ${ }^{* *} P<0.01$ versus $M C A O$.

fluoride, and pepstatin) and phosphatase inhibitors (Sigma cocktail, Sigma-Aldrich, St. Louis, MO, USA). For each sample, $100 \mu \mathrm{g}$ total protein was resolved by sodium dodecyl sulfate polyacrylamide gel electrophoresis, followed by electrophoretic transfer to polyvinylidene difluoride membranes. Membranes were incubated overnight at $4^{\circ} \mathrm{C}$ in a $1: 1000$ dilution of primary antibodies against caspase-3 (Abcam, Cambridge, UK), synaptophysin (Abcam), MAP-2 (Cell Signaling Technology, Boston, MA, USA), MBP (Abcam), brain derived neurotrophic factor (BDNF) (Abcam), or basic fibroblast growth factor (b-FGF) (Abcam). Chemiluminescent detection of antigens was performed following incubation with horseradish peroxidase-conjugated secondary antibodies (Santa Cruz Biotechnology, Santa Cruz, CA, USA) for $60 \mathrm{~min}$ at room temperature using an enhanced luminescence kit (Millipore, Billerica, MA, USA).

2.9. Immunofluorescence. Rats were euthanized 14 days after reperfusion with intraperitoneal injections of chloral hydrate
$(300 \mathrm{mg} / \mathrm{kg}$ ) and perfusion with cold saline. The brains were dehydrated in 30\% sucrose and $4 \%$ formaldehyde in PBS. Frozen brains were sectioned coronally for immunohistochemistry. Following incubation for $2 \mathrm{~h}$ in a blocking solution containing $1 \%$ bovine serum albumin, $2 \%$ normal goat serum, $0.3 \%$ Triton X-100, and $5 \%$ nonfat dry milk in PBS, sections were labelled using primary antibodies against synaptophysin (Abcam), MAP-2 (Cell Signaling Technology), MBP (Abcam), NF200 (Abcam), BDNF (Abcam), bFGF (Abcam), or neuron-specific nuclear protein (NeuN) (Millipore) at a dilution of 1:50. Sections were then incubated in fluorescently conjugated secondary antibodies (Alexa 488/Alexa 594-conjugated anti-mouse/anti-rabbit IgG) and fluorescence was detected using a fluorescence microscope (Carl Zeiss, Jena, Germany).

2.10. Statistical Analysis. Statistical analysis was performed using SPSS 11.0 (SPSS, Chicago, IL, USA). Data were expressed as means \pm SEM and were statistically analyzed by 


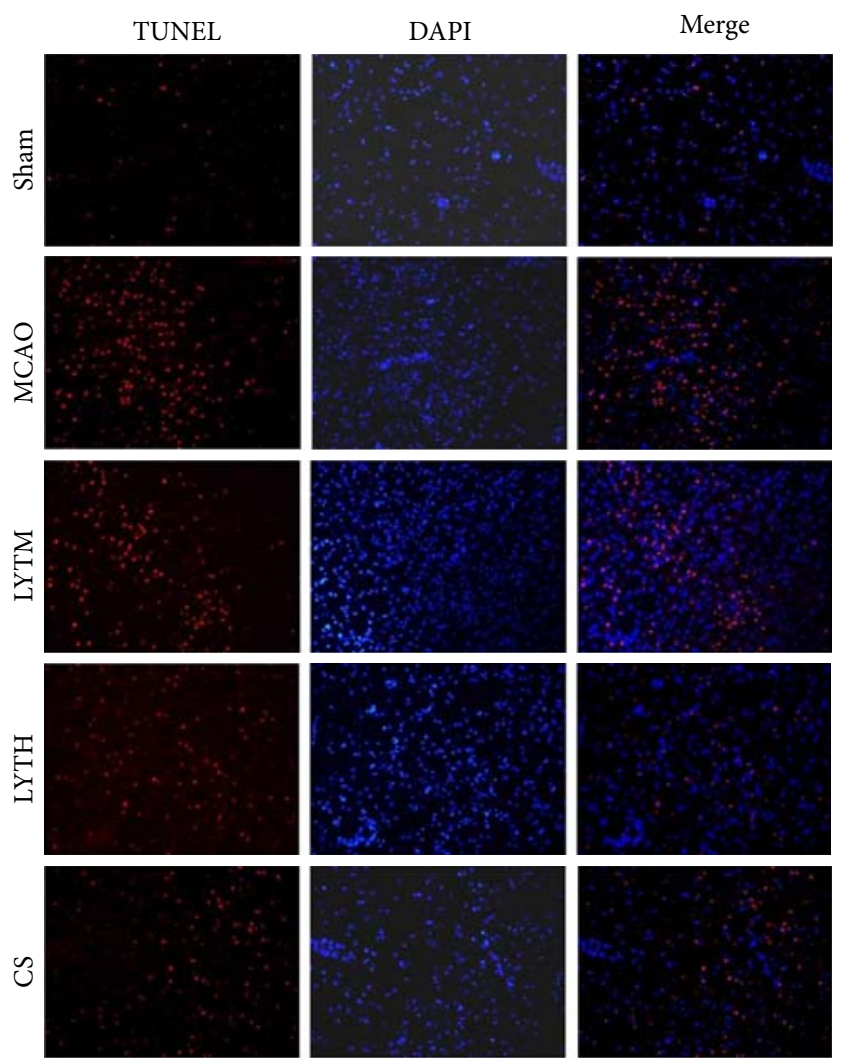

(a)
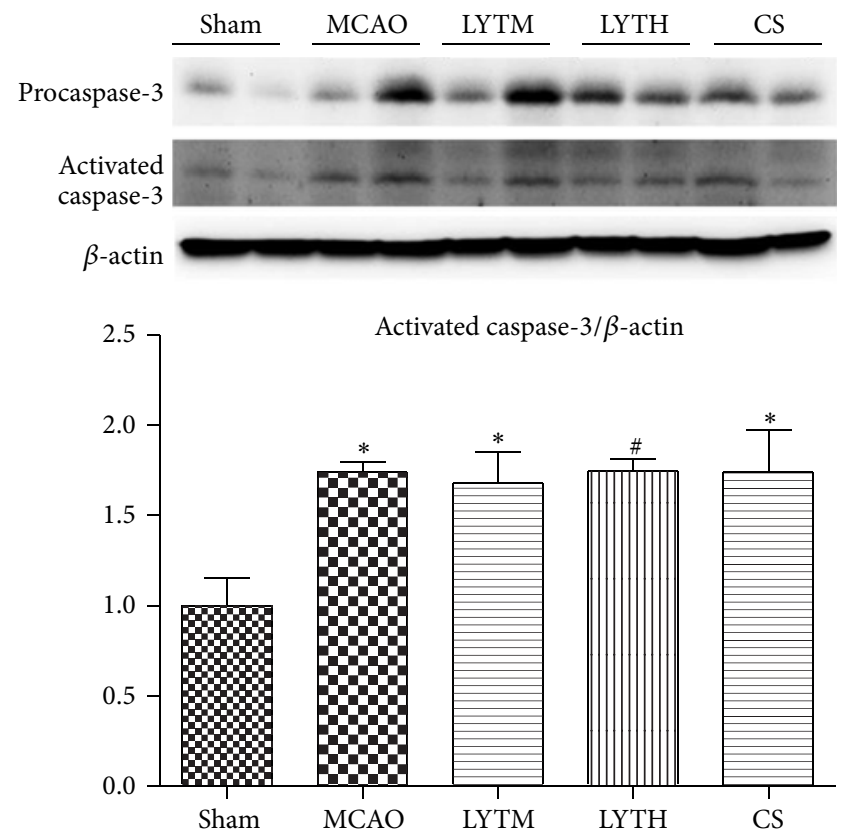

(b)

FIGURE 3: LYT (Luoyutong) reduces neuronal apoptosis and decreases the level of caspase-3. (a) Neuronal apoptosis in the ipsilateral cortex was detected by terminal deoxynucleotidyl transferase dUTP nick end labeling and $4^{\prime}$ 6-diamidino-2-phenylindole double staining 14 days after ischemic reperfusion. (b) Expression of activated caspase-3 was detected by Western blot 14 days after ischemic reperfusion. $n=3$. ${ }^{*} P<0.05$ versus Sham and ${ }^{\#} P<0.05$ versus $M C A O$.

one-way analysis of variance (ANOVA) followed by LSD post hoc test. $P$ values less than 0.05 were considered statistically significant.

\section{Results}

3.1. LYT Decreases Infarct Volume and Improves Neurological Function following Cerebral Ischemia-Reperfusion Injury in Rats. Behavioral tests and measurements of the infarct volume were used to evaluate the neurological outcome. The scores in all behavioral tests performed 3 days after reperfusion were not significantly different among groups (Figure 2(a)). There was a significant reduction in Longa's score test and the 0-12 neurological score test performance on day 14 in the LYTH group compared to the MCAO group (Longa's, $P<0.01 ; 0-12$ neurological score test, $P<$ $0.05)$, but the effect was not obvious in LYTM group and CS group, indicating improved neurological function in the LYTH group. Scores in the modified foot fault test were lower following all treatments (Figure 2(b)).

Cerebral infarct volume was measured by TTC staining (Figures 2(c) and 2(d)). The infarct volume in the LYTH group was significantly reduced compared to the MCAO group 3 and 14 days after ischemia-reperfusion injury (Figures 2(c) and 2(d), $P<0.05$ ). LYTM did not impact infarct volume as significantly as LYTH. CS treatment only decreased infarct volume after 14 days of treatment (Figure 2(d), $P<0.05$ ).

3.2. LYT Reduces Apoptosis in Neurons and Decreases the Level of Caspase-3. To measure apoptosis, TUNEL staining was performed in the cortex of ipsilateral cerebral tissue 14 days after reperfusion (Figure 3(a)). The number of apoptotic neurons in the MCAO group was significantly higher than that in the sham group. LYTH, LYTM, and CS treatment significantly decreased the number of TUNEL-positive cells compared to the MCAO group (Figure 3(a)). We also quantified the expression of activated caspase- 3 in cerebral tissue 14 days after reperfusion (Figure 3(b)). Activated caspase3 expression in the MCAO group was significantly higher than that in the sham group (Figure $3(\mathrm{~b}), P<0.05$ ). The expression of activated caspase-3 was reduced by LYTH, LTYM, and CS treatment, but a significant reduction was only observed in the LYTH group (Figure 3(b), $P<0.05$ ).

3.3. LYT Protects against Ischemia-Reperfusion Injury by Enhancing Neural Plasticity. To investigate the effect of LYT on neural plasticity following ischemia-reperfusion injury 


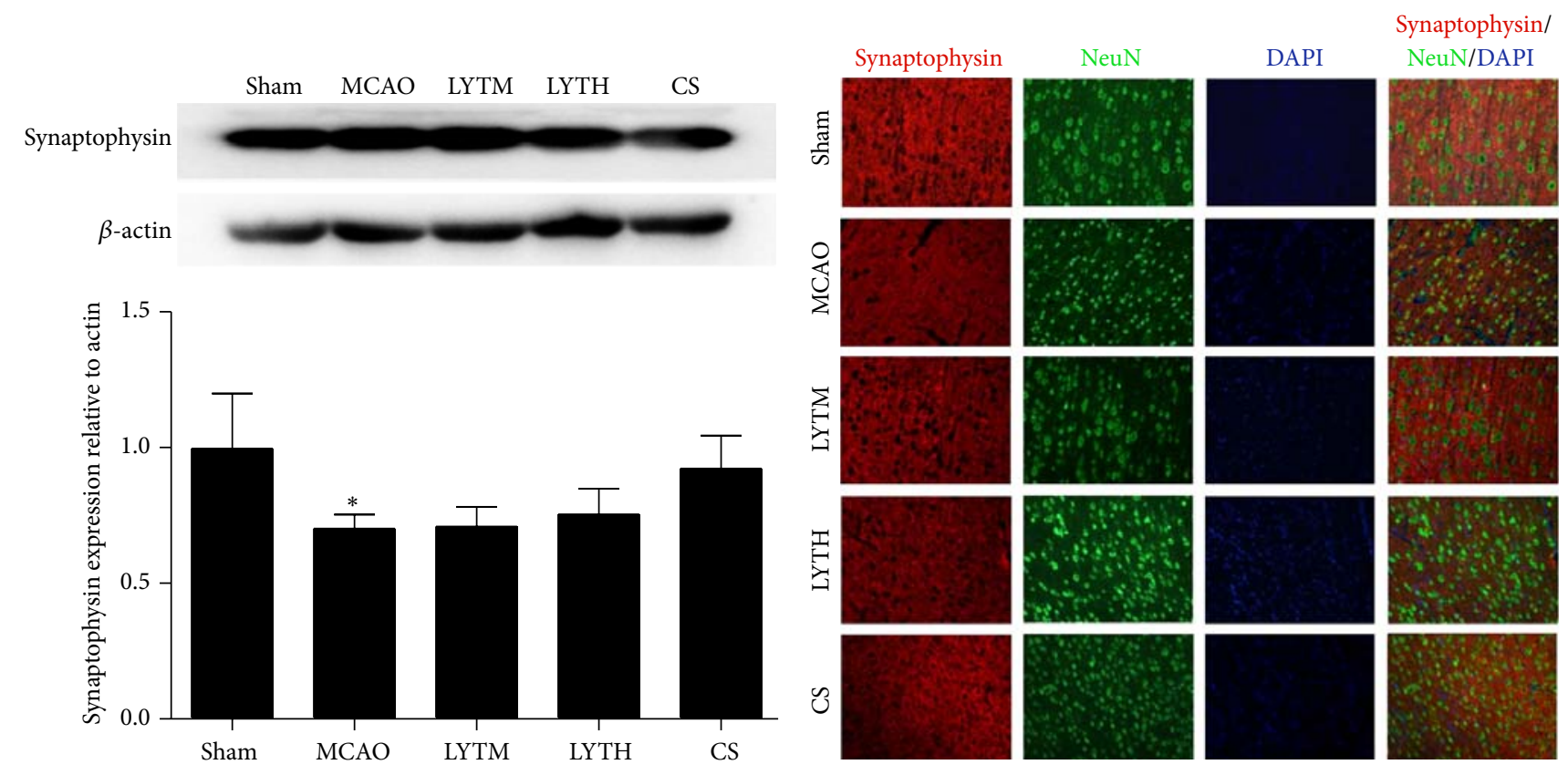

(a)

(b)

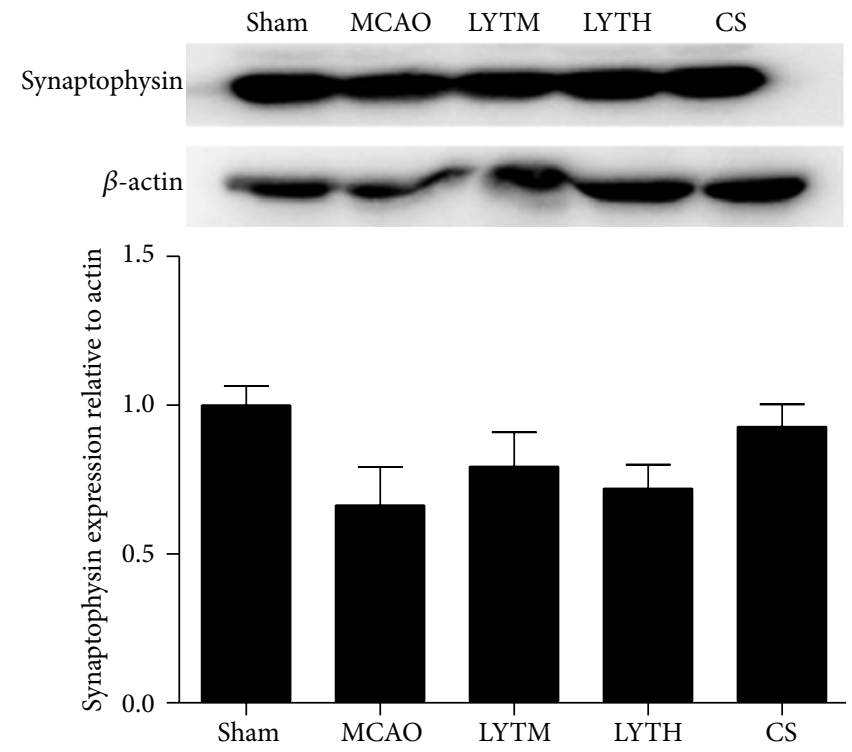

(c)

FIGURE 4: LYT (Luoyutong) induced upregulation of synaptophysin expression after MCAO (middle cerebral artery occlusion). Western blot detection and quantitative analysis of (a) synaptophysin expression 3 days after MCAO and (c) synaptophysin expression 14 days after MCAO. (b) Representative immunofluorescence images showing colocalization of synaptophysin (red) and NeuN (neuron-specific nuclear protein) (green) in the cortex. Blue DAPI staining indicates the nuclei. $n=3 .{ }^{*} P<0.05$ versus Sham.

in rats, we measured the levels of synaptophysin, MAP2, and MBP by Western blotting. We also analyzed the colocalization of synaptophysin and MAP-2 with NeuN in the cortex and MBP with NF 200 in the corpus callosum by immunofluorescence.

Synaptophysin expression was decreased in the MCAO group compared with the sham group 3 days after reperfusion (Figure 4(a), $P<0.05)$. Both of LYT and CS had no obvious effect on it (Figure 4(a), $P<0.05$ ). After 14 days, there were no significant differences between sham, MCAO, and treatment groups (Figure 4(c)). Immunofluorescence revealed that synaptophysin staining appeared as punctate-like or bouton-like distribution pattern with high immunofluorescence signal; staining of NeuN was shiny and sparkly. MCAO disrupted the localization of synaptophysin and dampened brightness of NeuN in the cortex, and this effect was reversed after 14 days of LYT and CS treatment (Figure 4(b)). 


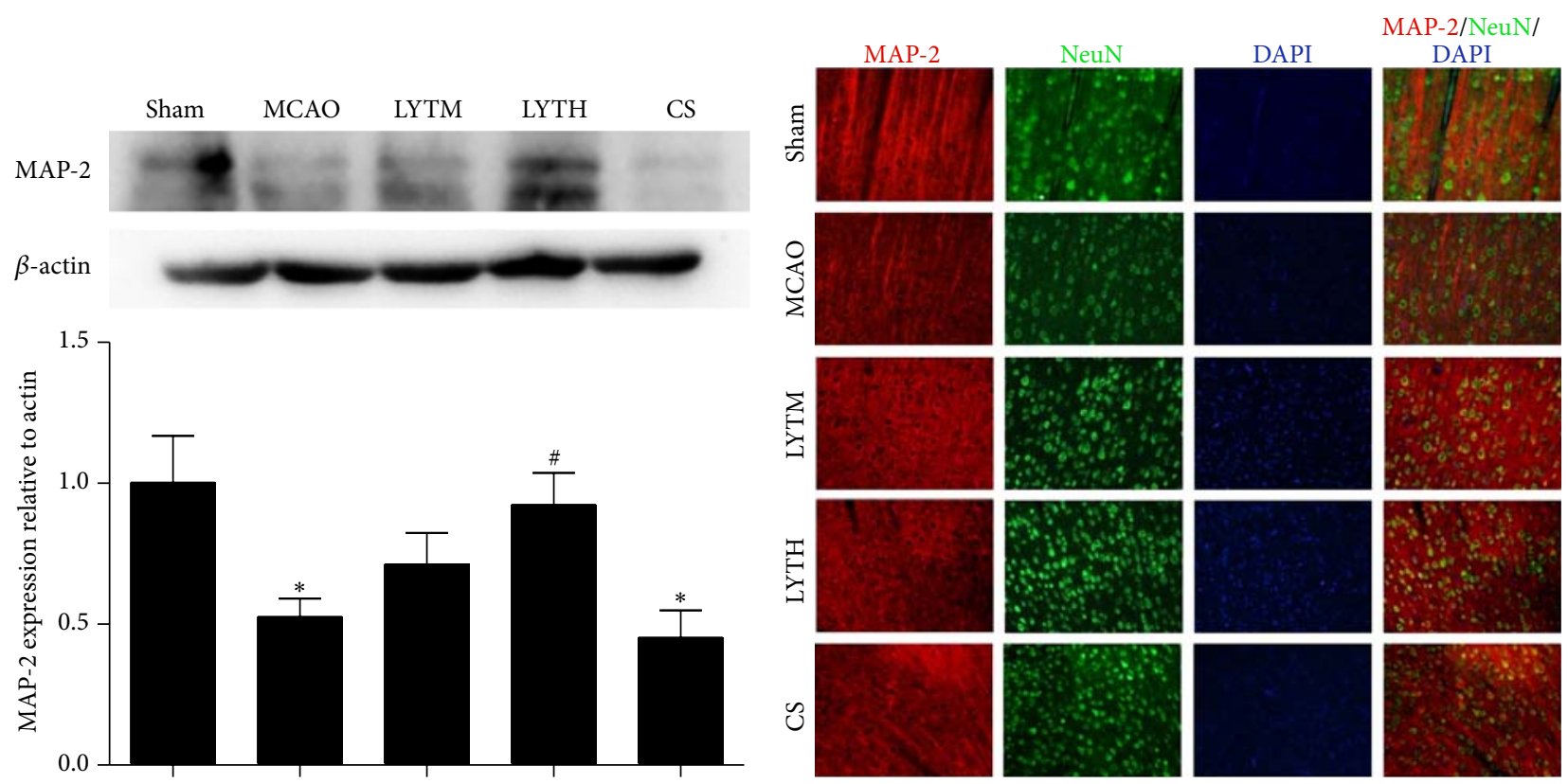

(a)

(b)
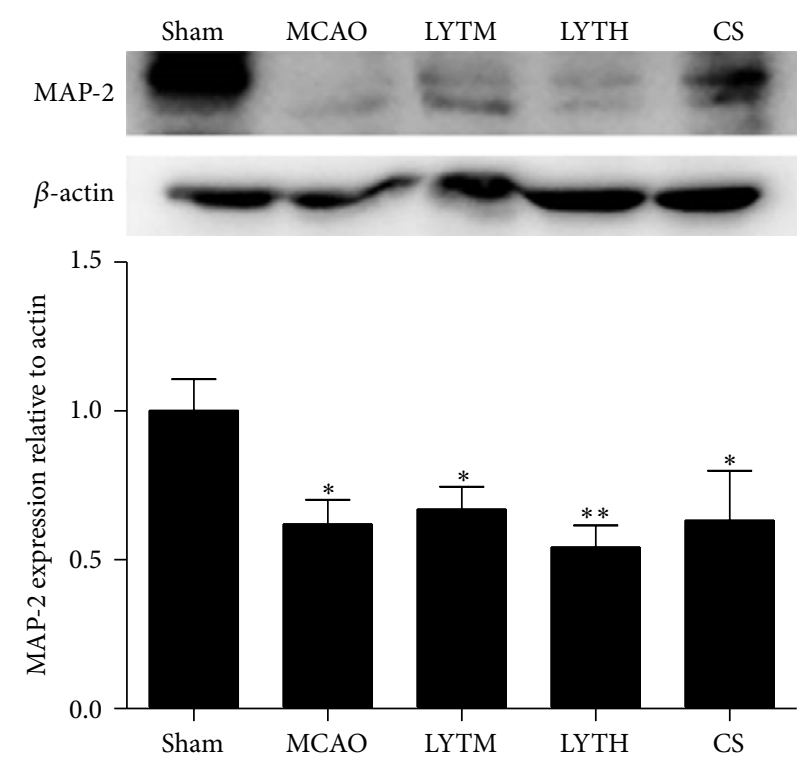

(c)

FIGURE 5: LYT (Luoyutong) induced upregulation of MAP-2 (microtubule associated protein) expression after MCAO (middle cerebral artery occlusion). Western blot detection and quantitative analysis of (a) MAP-2 expression 3 days after MCAO and (c) MAP-2 expression 14 days after MCAO. (b) Representative immunofluorescence images showing colocalization of MAP-2 (red) and NeuN (neuron-specific nuclear protein) (green) in the cortex. Blue DAPI staining indicates the nuclei. $n=3 .{ }^{*} P<0.05$ versus Sham, ${ }^{* *} P<0.01$ versus Sham, and ${ }^{\#} P<0.05$ versus MCAO.

Western blot analysis showed a reduced expression of MAP-2 in the MCAO group compared with the sham group 3 and 14 days after reperfusion (Figures 5(a) and 5(c), $P<0.05$ ). MAP-2 levels were increased by 3 days of LYTH treatment after reperfusion (Figure 5(a), $P<0.05$ ), whereas administration of LYTM and CA did not rescue MAP-2 expression. After 14 days, there was no difference between MCAO and treatment groups (Figure 5(c)). As shown in immunofluorescence, the expression of MAP-2 in sham group was shown in dendrites; it became irregular, discontinuous, and weaker in MCAO and it was improved by LYTH and LYTM treatment but not CS treatment (Figure 5(b)).

The expression of MBP was lower in the MCAO group compared to the sham group 3 and 14 days after reperfusion, but this was only statistically significant after 14 days (Figures 6(a) and 6(c), $P<0.01)$. We observed an increased MBP expression after 3 and 14 days of treatment with LYT and CS (Figures 6(a) and 6(c)); similarly the significant 


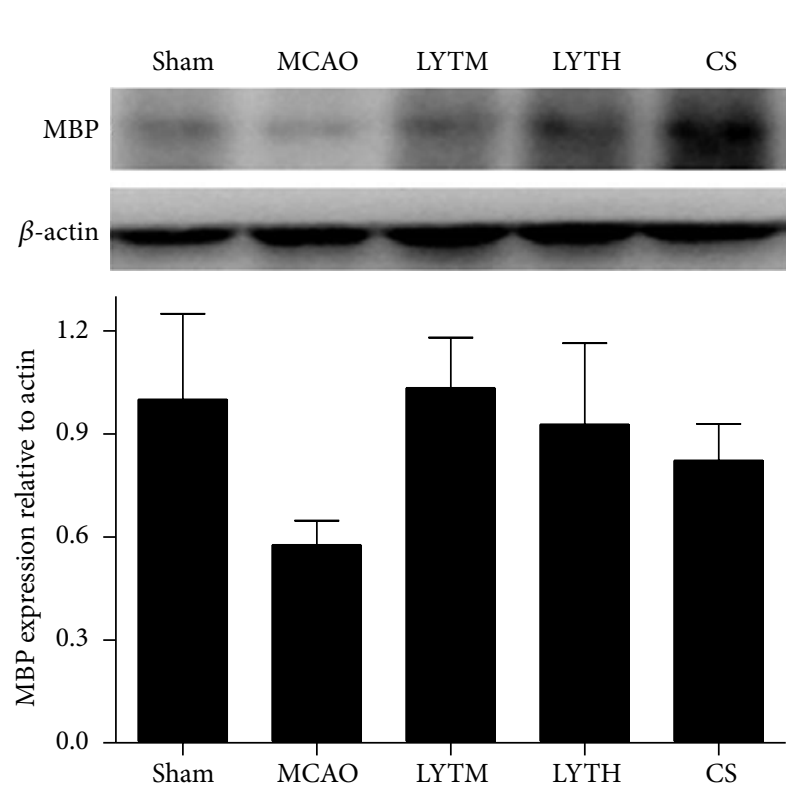

(a)
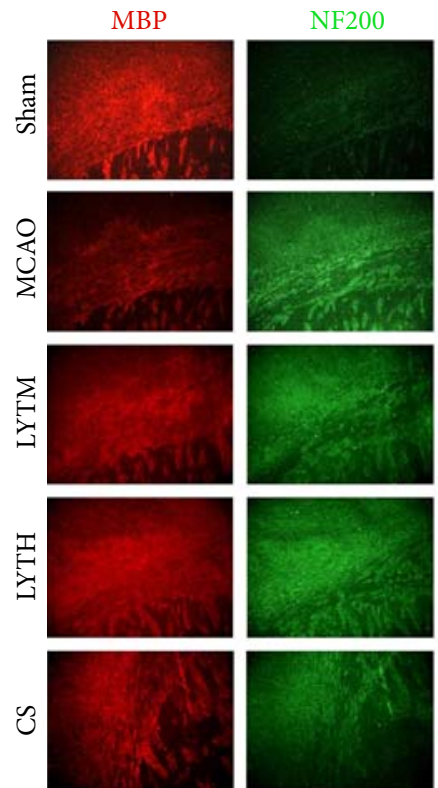

(b)
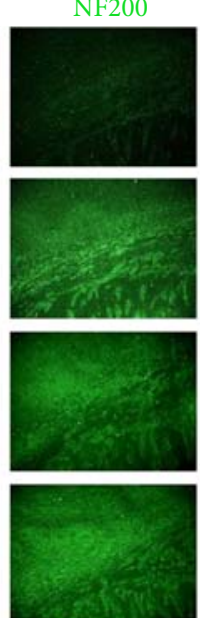

MBP/NF200/
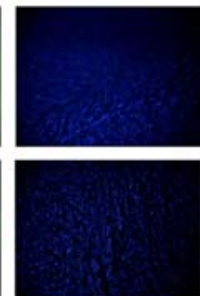

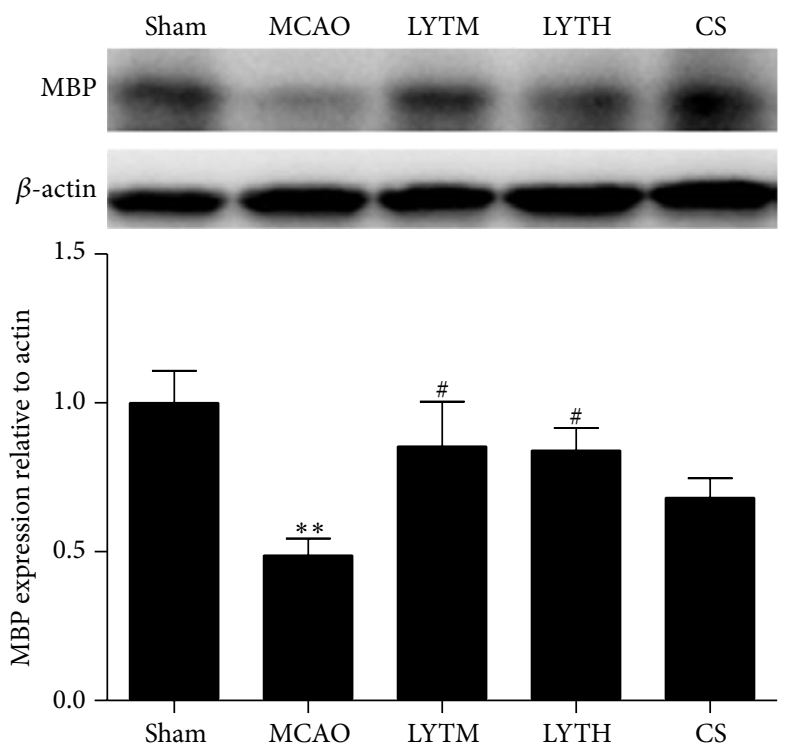

(c)

FIGURE 6: LYT (Luoyutong) induced upregulation of MBP (myelin basic protein) expression after MCAO (middle cerebral artery occlusion). Western blot detection and quantitative analysis of (a) MBP expression 3 days after MCAO and (c) MBP expression 14 days after MCAO. (b) Representative immunofluorescence images showing colocalization of MBP (red) and NF200 (neurofilament 200) (green) in the cortex. Blue DAPI staining indicates the nuclei. $n=3$. ${ }^{* *} P<0.01$ versus Sham and ${ }^{\#} P<0.05$ versus MCAO.

difference was only significant after 14 days of LYT treatment (Figure 6(c), $P<0.05$ ). MBP immunoreactivity was affected mostly by myelin sheaths in the sham group. However, low immunoreactivity was detected for MBP after MCAO, while NF200 staining increased in intensity in the MCAO group. These effects were reversed by all treatments (Figure 6(b)).

Taken together, these findings demonstrated that the different treatments protected neural plasticity following ischemia-reperfusion injury, particularly the high dose of LYT.
3.4. LYT Treatment Upregulated BDNF and b-FGF after Ischemia-Reperfusion Injury. To investigate the effect of LYT treatment on the expression of growth factors, we measured BDNF and b-FGF expressions by Western blot 3 and 14 days after reperfusion. BDNF expression was significantly lower in the MCAO group compared to the sham group 3 days after reperfusion (Figure $7(\mathrm{a}), P<0.05$ ). LYT and CS treatment dramatically increased BDNF expression 3 days after reperfusion and it was even higher than that in the sham group (Figure $7(\mathrm{a}), P<0.05$ ). After 14 days, no 


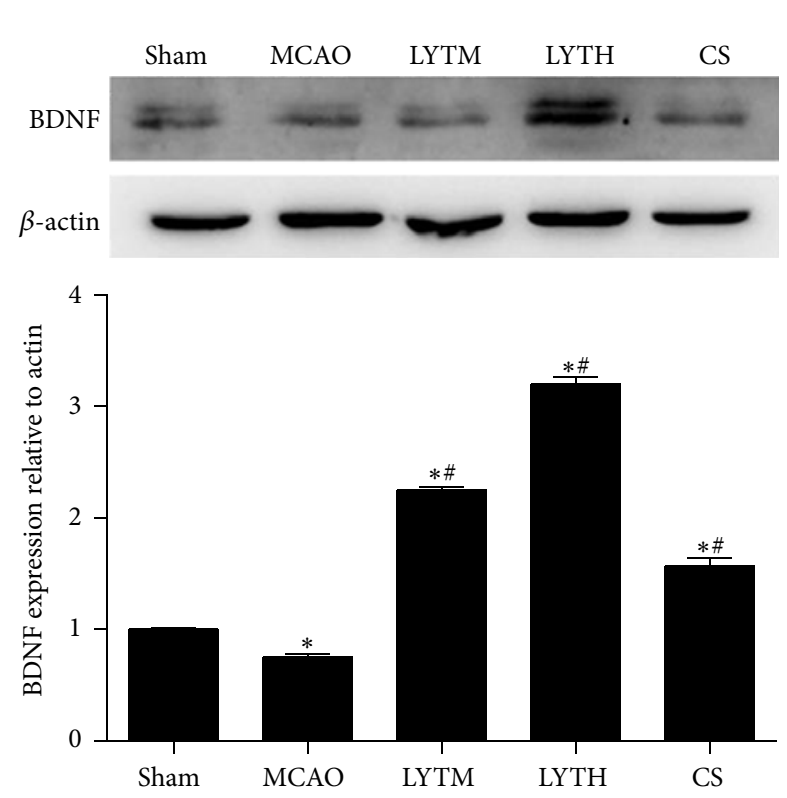

(a)
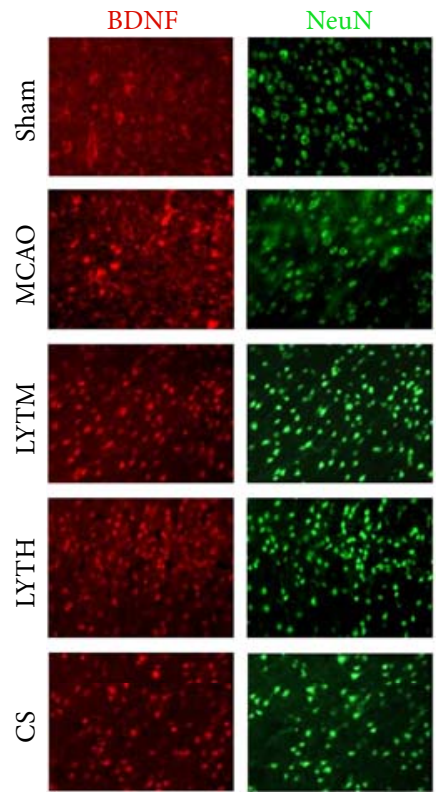

(b)

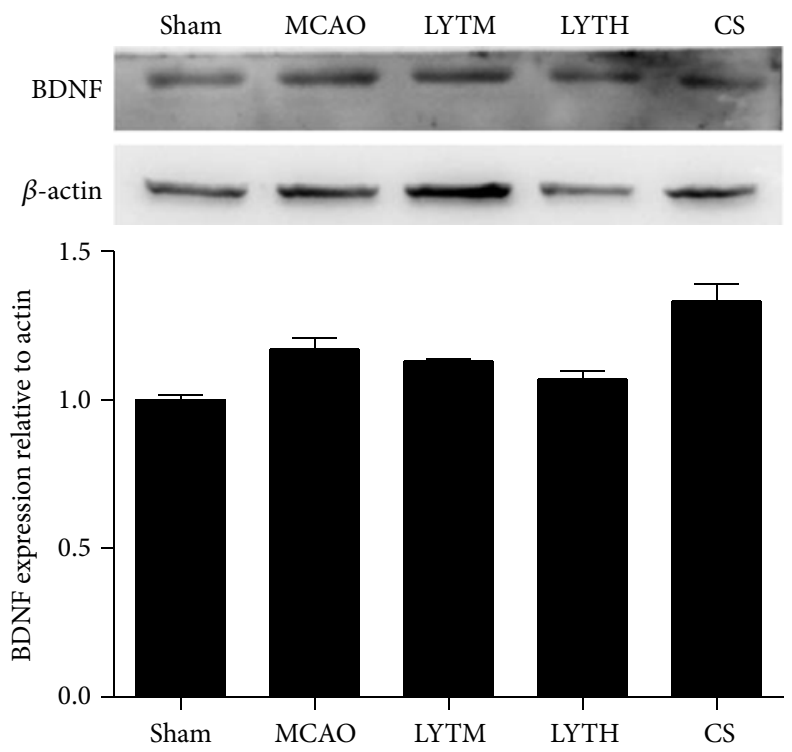

(c)

FIGURE 7: LYT (Luoyutong) induced upregulation of BDNF (brain derived neurotrophic factor) expression after MCAO (middle cerebral artery occlusion). Western blot detection and quantitative analysis of (a) BDNF expression 3 days after MCAO and (c) BDNF expression 14 days after MCAO. (b) Representative immunofluorescence images showing colocalization of BDNF (red) and NeuN (neuron-specific nuclear protein) (green) in the cortex. Blue DAPI staining indicates the nuclei. $n=3 .{ }^{* *} P<0.01$ versus Sham and ${ }^{\#} P<0.05$ versus $M C A O$.

significant differences were observed in BDNF expression between the groups (Figure 7(c)). BDNF positive cells partly colocalized with NeuN as demonstrated by immunofluorescence; the staining of BDNF was not different among groups (Figure 7(b)).

b-FGF expression was also lower in the MCAO group compared to the sham group 3 days after reperfusion (Figure $8(\mathrm{a}), P<0.05$ ) and only treatment with LYTH could reverse this effect. No difference in b-FGF expression was observed 14 days after reperfusion (Figure 8(c)). Immunofluorescence revealed that a portion of b-FGF showed colocalization with NeuN in the cortex; MCAO and treatment had no effect on its expression (Figure 8(b)).

\section{Discussion}

Clinical research showed that LYT has a therapeutic effect of stroke [12, 13]; however, the mechanism underlying the 


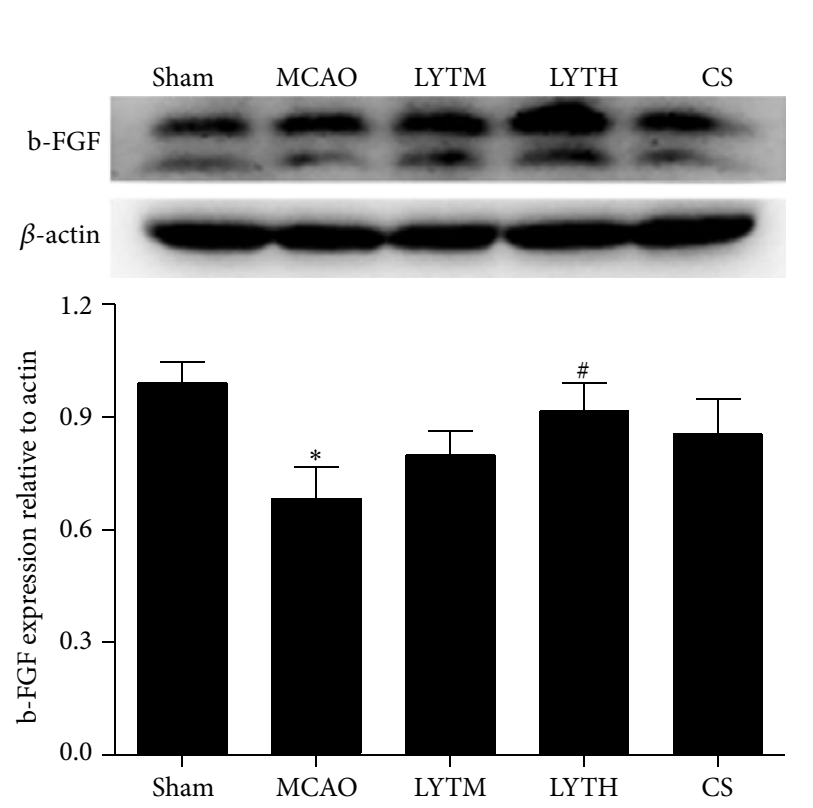

(a)
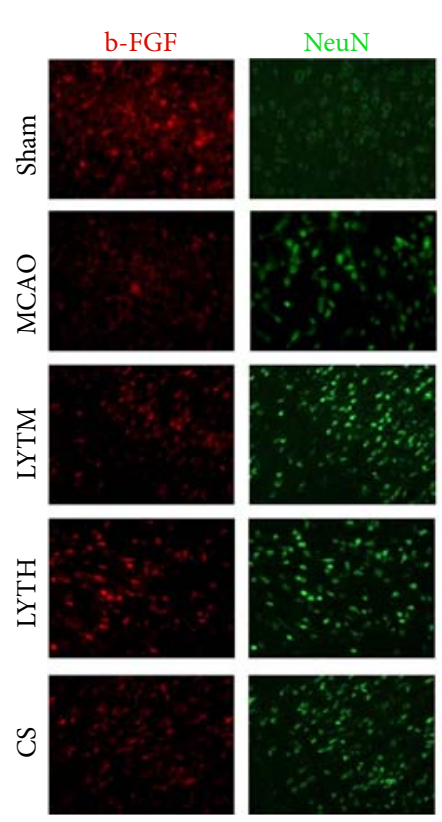

(b)
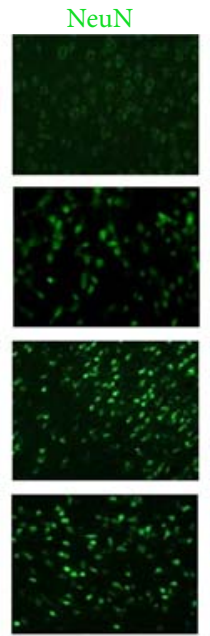

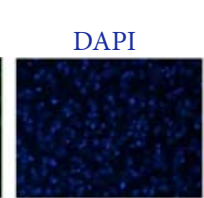

b-FGF/NeuN/
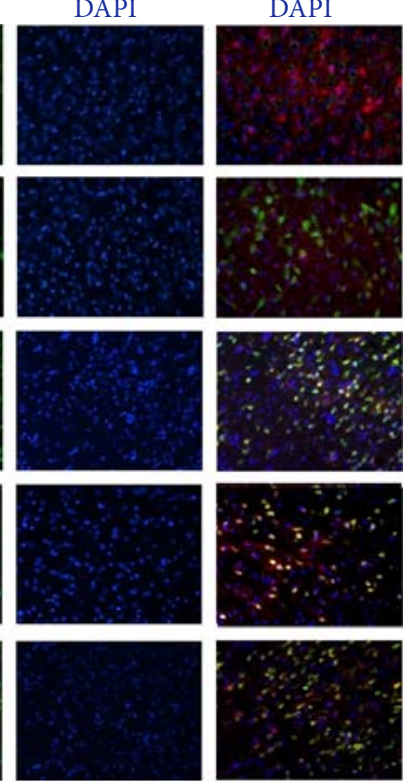
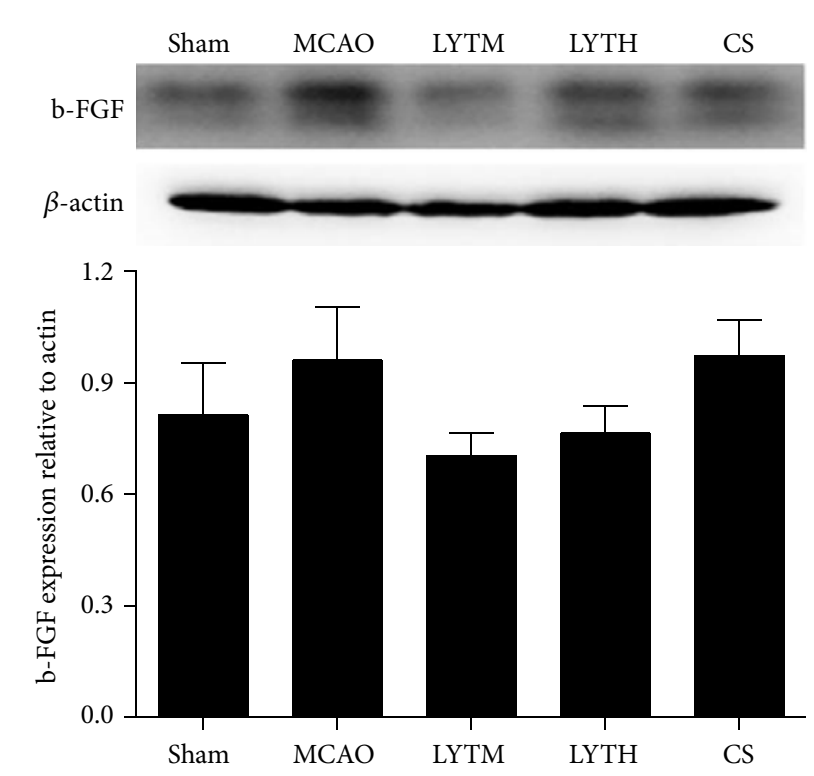

(c)

FIGURE 8: LYT (Luoyutong) induced upregulation of b-FGF (basic fibroblast growth factor) expression after MCAO (middle cerebral artery occlusion). Western blot detection and quantitative analysis of (a) b-FGF expression 3 days after MCAO and (c) b-FGF expression 14 days after MCAO. (b) Representative immunofluorescence images showing colocalization of b-FGF (red) and NeuN (neuron-specific nuclear protein) (green) in the cortex. Blue DAPI staining indicates the nuclei. $n=3 .{ }^{* *} P<0.01$ versus Sham and ${ }^{\#} P<0.05$ versus $M C A O$.

therapeutic effects remains undefined. To address this, LYT was applied to rat model of cerebral ischemia-reperfusion injury. As expected, LYTH treatment could improve neurologic function and reduce infarction volume, which is more significant than CS treatment group. Further studies revealed that the protective effect of LYT is potentially related to the improvement of neural plasticity and upregulation of BDNF and b-FGF. The measurement of infarction volume showed that LYT reduces infarct volume significantly in both acute stage $(3 \mathrm{~d})$ and restoration stage $(14 \mathrm{~d})$. It is noteworthy that CS has no effect on it in acute stage $(3 \mathrm{~d})$. Although CS is widely used in clinic, its treatment still has limitations. Moreover, LYT showed the improvement effect of neurological function at 14 days after ischemia-reperfusion injury in 
accord with clinical research $[12,13]$. We speculate that early inhibition of infarction volume is more advantageous to the late reply of neurological function.

In order to make clear whether the therapeutic effect of LYT depends on antiapoptosis function, we measured apoptosis by TUNEL and quantified the expression of activated caspase- 3 in cerebral tissue 14 days after reperfusion. Both CS and LYTH have pronounced influence on apoptosis, but only LYTH could reduce the expression of activated caspase3. Since caspase-3 is not only a known regulator of apoptosis, it can also influence neural plasticity [19]. Therefore, the downregulation of caspase- 3 in response to LYT treatment not only may influence apoptosis exclusively but also can promote neural plasticity.

Neural plasticity is important for neurological function recovery after reperfusion, and neural plasticity has different performance at different time points. Neural plasticity plays a vital role in ischemic injury [20], depression, and memory [21, 22]. MAP-2 is dendritic marker and synaptophysin is synaptic marker. Synaptophysin regulates activity-dependent synapse formation [23]. MAP-2 nucleates and stabilizes microtubules, regulates organelle transport, and anchors regulatory proteins within neurons to regulate process outgrowth, synaptic plasticity, and apoptosis [24]. In present study, our results demonstrated that synaptophysin and MAP-2 levels decrease after MCAO, in agreement with previous findings [25, 26]. Meanwhile MCAO disorganized the arrangement structure of MAP-2, but the arrangement structure is the foundation of its function. The result showed that only LYTH could increase the level of MAP-2, and this function only embodied in 3 days. CS and LYTM do not have obvious effect on it. But all of the treatments have no effect on synaptophysin. These findings suggest that LYTH can enhance neural plasticity through regulating the expression of MAP-2 following ischemiareperfusion injury in gray matter. Cerebral white matter is also highly vulnerable to ischemia, and white matter is subject to a novel form of neural plasticity which is termed "myelin plasticity" [27]. Changes in MBP and NF200 expression can be indicative of white matter injury following MCAO [28]. The present study is consistent with previous reports on MBP and NF200 expression changes after ischemia-reperfusion injury. After 14 days, the change is more prominent. LYT treatment can reverse these effects and the improvement of LYT is more remarkable after 14 days. However, the effect of CS is not obvious. Our results revealed a regulatory role of LYT in white matter plasticity following cerebral ischemiareperfusion injury, while CS treatment could not improve white matter injury.

In addition, LYT treatment upregulates the expression of BDNF and b-FGF following ischemia-reperfusion injury, both of which are critical mediators of neural plasticity and survival $[19,29-35]$. The influence of MCAO and the intervention effect of treatment only reflect on the third day. The results from the expression of BNDF and b-FGF indicate that LYT is stronger than CS in regulating growth factors, especially LYTH.

In summary, we have provided evidence that LYT has a protective effect after ischemia-reperfusion injury in rats by suppressing neuronal apoptosis and repairing neural plasticity of both gray matter and white matter. These effects may be mediated by regulating caspase-3, BDNF, and b-FGF expressions. Further elucidation of the signaling pathways involved is required. It is likely that the herbal ingredients of LYT act synergistically, but the relative contribution of each herb to the beneficial outcome has not yet been defined.

\section{Disclosure}

Ning-qun Wang and Li-ye Wang are co-first authors.

\section{Conflict of Interests}

The authors declare that there is no conflict of interests regarding the publication of this paper.

\section{Acknowledgments}

This work was supported by Projects of Beijing Nova Program (Grant no. Z151100000315065) and Chinese Natural Science Foundation grants (Grant nos. 81271461, 81571280, and 81325007).

\section{References}

[1] R. S. Pandya, L. Mao, H. Zhou et al., "Central nervous system agents for ischemic stroke: neuroprotection mechanisms," Central Nervous System Agents in Medicinal Chemistry, vol. 11, no. 2, pp. 81-97, 2011.

[2] K. Sun, Q. Hu, C. M. Zhou et al., "Cerebralcare Granule, a Chinese herb compound preparation, improves cerebral microcirculatory disorder and hippocampal CA1 neuron injury in gerbils after ischemia-reperfusion," Journal of Ethnopharmacology, vol. 130, no. 2, pp. 398-406, 2010.

[3] Z.-J. Zhang, P. Li, Z. Wang et al., "A comparative study on the individual and combined effects of baicalin and jasminoidin on focal cerebral ischemia-reperfusion injury," Brain Research, vol. 1123, no. 1, pp. 188-195, 2006.

[4] Y. He, H. Wan, Y. Du et al., "Protective effect of Danhong injection on cerebral ischemia-reperfusion injury in rats," Journal of Ethnopharmacology, vol. 144, no. 2, pp. 387-394, 2012.

[5] Z. H. Lin, D. N. Zhu, Y. Q. Yan, and B. Yu, "Herbal formula FBD extracts prevented brain injury and inflammation induced by cerebral ischemia-reperfusion," Journal of Ethnopharmacology, vol. 118, no. 1, pp. 140-147, 2008.

[6] L.-D. Zhao, J.-H. Wang, G.-R. Jin, Y. Zhao, and H.-J. Zhang, "Neuroprotective effect of Buyang Huanwu decoction against focal cerebral ischemia/reperfusion injury in rats-time window and mechanism," Journal of Ethnopharmacology, vol. 140, no. 2, pp. 339-344, 2012.

[7] M. H. Cohen and K. J. Kemper, "Complementary therapies in pediatrics: a legal perspective," Pediatrics, vol. 115, no. 3, pp. 774780, 2005.

[8] C.-C. Shih, C.-C. Liao, Y.-C. Su, C.-C. Tsai, and J.-G. Lin, "Gender differences in traditional chinese medicine use among adults in Taiwan," PLoS ONE, vol. 7, no. 4, Article ID e32540, 2012.

[9] V. Chung, E. Wong, J. Woo, S. V. Lo, and S. Griffiths, "Use of traditional Chinese medicine in the Hong Kong special 
administrative region of China," Journal of Alternative and Complementary Medicine, vol. 13, no. 3, pp. 361-367, 2007.

[10] Z. Junhua, F. Menniti-Ippolito, G. Xiumei et al., "Complex traditional Chinese medicine for poststroke motor dysfunction: a systematic review," Stroke, vol. 40, no. 8, pp. 2797-2804, 2009.

[11] C. Matute, M. Domercq, A. Pérez-Samartín, and B. R. Ransom, "Protecting white matter from stroke injury," Stroke, vol. 44, no. 4, pp. 1204-1211, 2013.

[12] J. F. Wan, X. N. Zhang, J. Q. Guo, and C. F. Wang, "Clinical efficacy and safety evaluation of Luoyutong capsule treating on stroke," Asia-Pacific Traditional Medicine, vol. 10, no. 7, pp. 106107, 2014.

[13] T. S. Liu, S. Zhou, D. H. Wu et al., "Summary of 99 patients used Luoyutong capsule to treating stroke (cerebral thrombosis)," Geriatrics Health Care, vol. 10, no. 4, p. 243, 2004.

[14] L.-Y. Wang, H.-P. Zhao, R.-H. Wang et al., "Effect of Luoyutong capsule on focal cerebral ischemia-reperfusion injury in rats," Chinese Journal of Cerebrovascular Diseases, vol. 11, no. 12, pp. 650-655, 2014.

[15] E. Z. Longa, P. R. Weinstein, S. Carlson, and R. Cummins, "Reversible middle cerebral artery occlusion without craniectomy in rats," Stroke, vol. 20, no. 1, pp. 84-91, 1989.

[16] S. J. Kim, B. K. Kim, Y. J. Ko, M. S. Bang, M. H. Kim, and T. R. Han, "Functional and histologic changes after repeated transcranial direct current stimulation in rat stroke model," Journal of Korean Medical Science, vol. 25, no. 10, pp. 1499-1505, 2010.

[17] D. C. Rogers, C. A. Campbell, J. L. Stretton, and K. B. Mackay, "Correlation between motor impairment and infarct volume after permanent and transient middle cerebral artery occlusion in the rat," Stroke, vol. 28, no. 10, pp. 2060-2066, 1997.

[18] J. M. Mountz, "Measuring brain infarct volume," Journal of Cerebral Blood Flow \& Metabolism, vol. 11, no. 1, p. 168, 1991.

[19] Z. Li, J. Jo, J.-M. Jia et al., "Caspase-3 activation via mitochondria is required for long-term depression and AMPA receptor internalization," Cell, vol. 141, no. 5, pp. 859-871, 2010.

[20] T. H. Murphy, "Two-photon imaging of neuronal structural plasticity in mice during and after ischemia," Cold Spring Harb Protoc, no. 6, pp. 548-557, 2015.

[21] C. L. Busceti, P. D. Pietro, B. Riozzi et al., " $5-\mathrm{HT}_{2 \mathrm{C}}$ serotonin receptor blockade prevents tau protein hyperphosphorylation and corrects the defect in hippocampal synaptic plasticity caused by a combination of environmental stressors in mice," Pharmacological Research, vol. 99, pp. 258-268, 2015.

[22] E. Castrén and R. Hen, "Neuronal plasticity and antidepressant actions," Trends in Neurosciences, vol. 36, no. 5, pp. 259-267, 2013.

[23] L. Tarsa and Y. Goda, "Synaptophysin regulates activitydependent synapse formation in cultured hippocampal neurons," Proceedings of the National Academy of Sciences of the United States of America, vol. 99, no. 2, pp. 1012-1016, 2002.

[24] C. Sánchez, J. Díaz-Nido, and J. Avila, "Phosphorylation of microtubule-associated protein 2 (MAP2) and its relevance for the regulation of the neuronal cytoskeleton function," Progress in Neurobiology, vol. 61, no. 2, pp. 133-168, 2000.

[25] F. D. Pinheiro Fernandes, A. P. Fontenele Menezes, J. C. de Sousa Neves et al., "Caffeic acid protects mice from memory deficits induced by focal cerebral ischemia," Behavioural Pharmacology, vol. 25, no. 7, pp. 637-647, 2014.

[26] L. C. Yang, J. Li, S. F. Xu et al., "L-3-n-butylphthalide promotes neurogenesis and neuroplasticity in cerebral ischemic rats,"
CNS Neuroscience \& Therapeutics, vol. 21, no. 9, pp. 733-741, 2015.

[27] S. Rosenzweig and S. T. Carmichael, "Age-dependent exacerbation of white matter stroke outcomes: a role for oxidative damage and inflammatory mediators," Stroke, vol. 44, no. 9, pp. 2579-2586, 2013.

[28] J. Suenaga, X. Hu, H. Pu et al., "White matter injury and microglia/macrophage polarization are strongly linked with age-related long-term deficits in neurological function after stroke," Experimental Neurology, vol. 272, pp. 109-119, 2015.

[29] M.-M. Poo, "Neurotrophins as synaptic modulators," Nature Reviews Neuroscience, vol. 2, no. 1, pp. 24-32, 2001.

[30] R. S. Duman and B. Voleti, "Signaling pathways underlying the pathophysiology and treatment of depression: novel mechanisms for rapid-acting agents," Trends in Neurosciences, vol. 35, no. 1, pp. 47-56, 2012.

[31] V. Duric, M. Banasr, P. Licznerski et al., "A negative regulator of MAP kinase causes depressive behavior," Nature Medicine, vol. 16, no. 11, pp. 1328-1332, 2010.

[32] G. L. Collingridge, S. Peineau, J. G. Howland, and Y. T. Wang, "Long-term depression in the CNS," Nature Reviews Neuroscience, vol. 11, no. 7, pp. 459-473, 2010.

[33] B. Lu, "BDNF and activity-dependent synaptic modulation," Learning and Memory, vol. 10, no. 2, pp. 86-98, 2003.

[34] S. Yang and J. Cui, "Study on the value of exogenous bFGF in the treatment of brain injury," Chinese Journal of Traumatology, vol. 3, no. 3, pp. 131-135, 2000.

[35] K. Chaiyasate, A. Schaffner, I. T. Jackson, and V. Mittal, “Comparing FK-506 with basic fibroblast growth factor (b-FGF) on the repair of a peripheral nerve defect using an autogenous vein bridge model," Journal of Investigative Surgery, vol. 22, no. 6, pp. 401-405, 2009. 


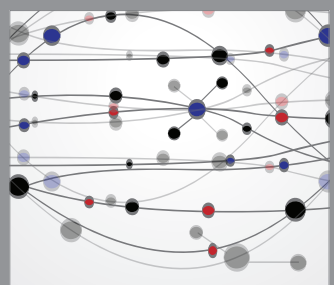

The Scientific World Journal
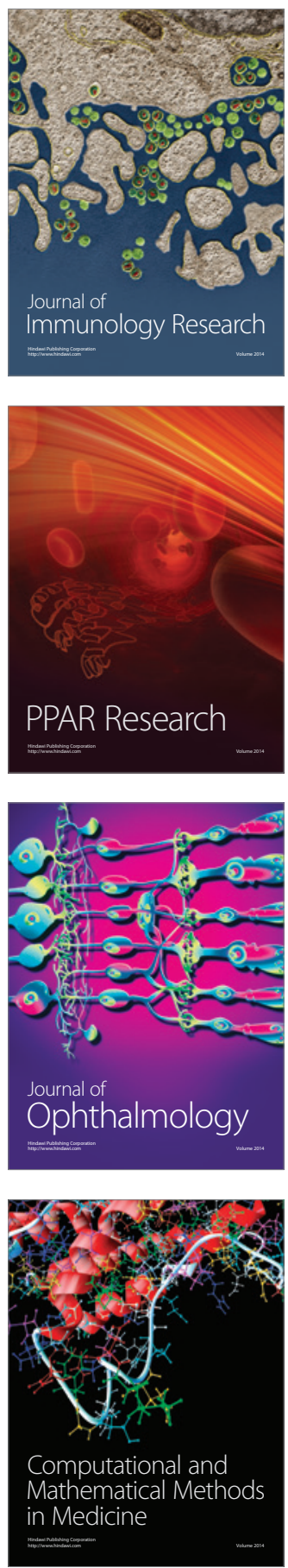

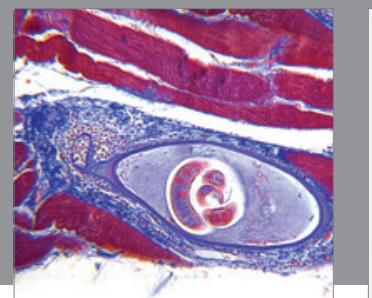

Gastroenterology

Research and Practice
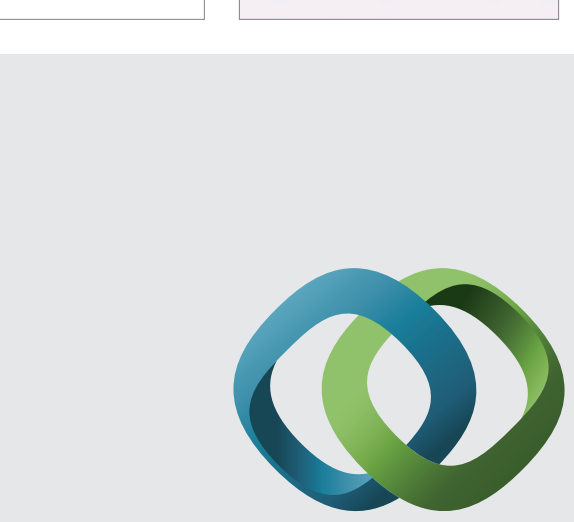

\section{Hindawi}

Submit your manuscripts at

http://www.hindawi.com
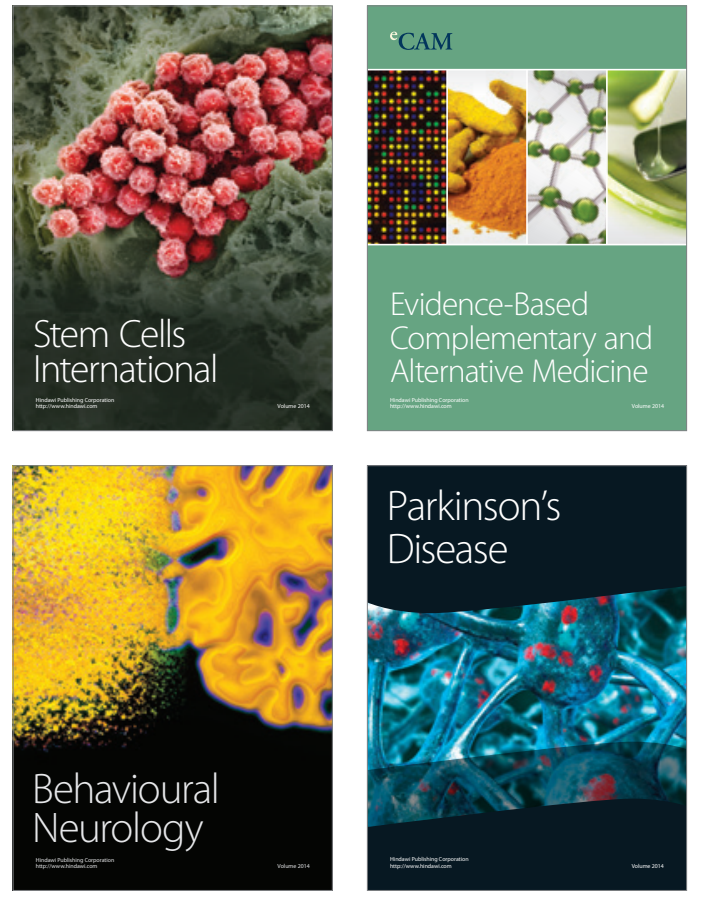
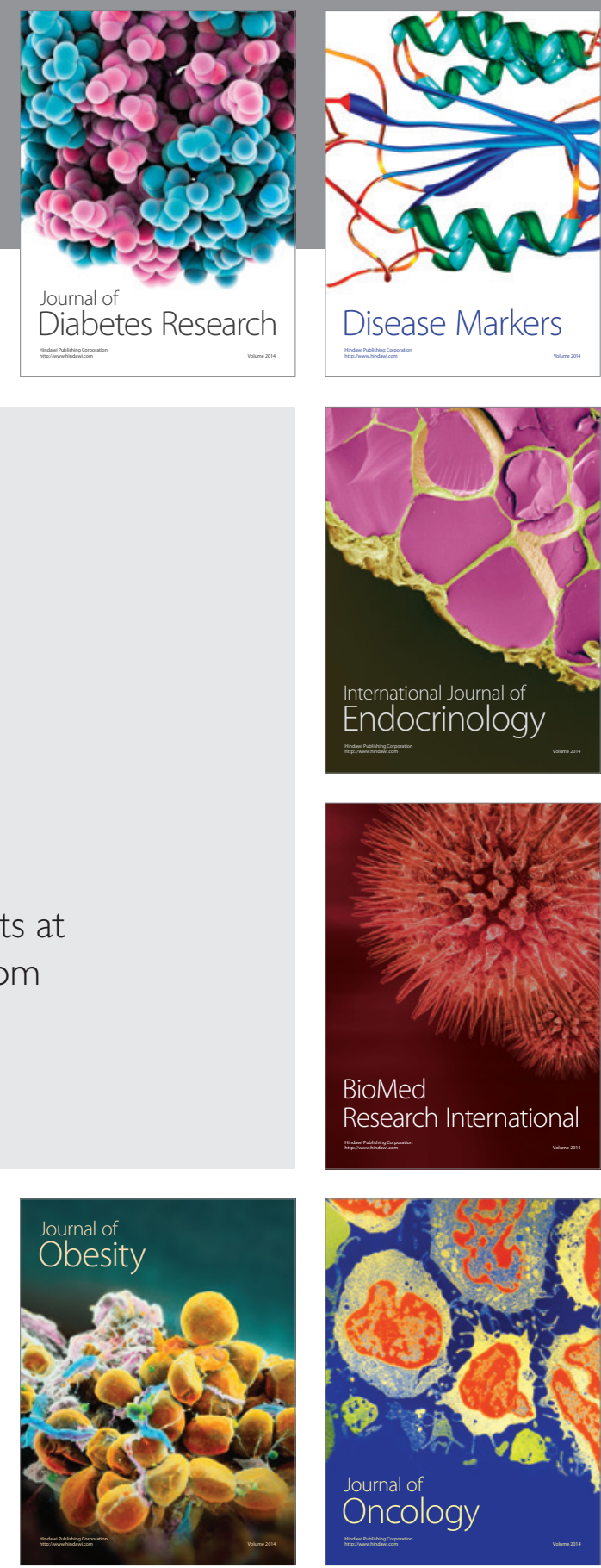

Disease Markers
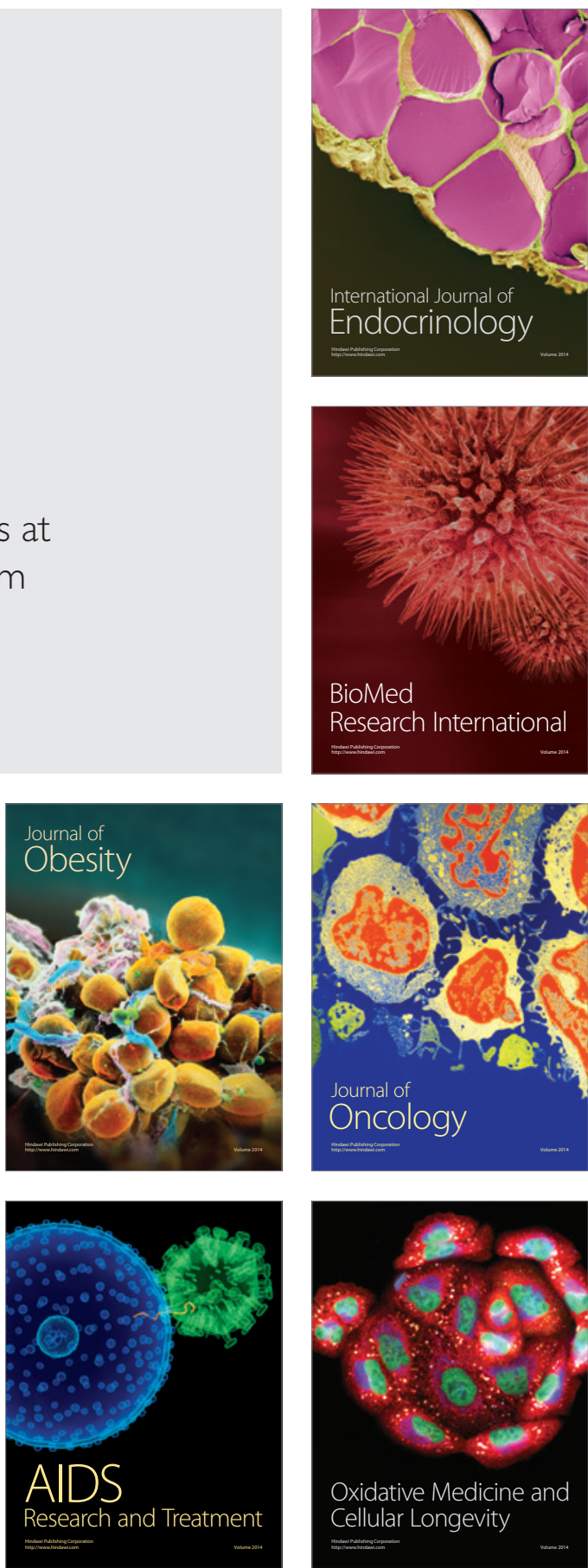